\title{
Diagnosis and management of primary aldosteronism
}

Leticia A. P. Vilela', Madson Q. Almeida',

\begin{abstract}
Primary aldosteronism (PA) is the most common form of secondary hypertension (HTN), with an estimated prevalence of $4 \%$ of hypertensive patients in primary care and around $10 \%$ of referred patients. Patients with PA have higher cardiovascular morbidity and mortality than age- and sexmatched patients with essential HTN and the same degree of blood pressure elevation. PA is characterized by an autonomous aldosterone production causing sodium retention, plasma renin supression, HTN, cardiovascular damage, and increased potassium excretion, leading to variable degrees of hypokalemia. Aldosterone-producing adenomas (APAs) account for around $40 \%$ and idiopathic hyperaldosteronism for around $60 \%$ of PA cases. The aldosterone-to-renin ratio is the most sensitive screening test for PA. There are several confirmatory tests and the current literature does not identify a "gold standard" confirmatory test for PA. In our institution, we recommend starting case confirmation with the furosemide test. After case confirmation, all patients with PA should undergo adrenal CT as the initial study in subtype testing to exclude adrenocortical carcinoma. Bilateral adrenal vein sampling (AVS) is the gold standard method to define the PA subtype, but it is not indicated in all cases. An experienced radiologist must perform AVS. Unilateral laparoscopic adrenalectomy is the preferential treatment for patients with APAs, and bilateral hyperplasia should be treated with mineralocorticoid antagonist (spironolactone or eplerenone). Cardiovascular morbidity caused by aldosterone excess can be decreased by either unilateral adrenalectomy or mineralocorticoid antagonist. In this review, we address the most relevant issues regarding PA screening, case confirmation, subtype classification, and treatment. Arch Endocrinol Metab. 2017;61(3):305-12.
\end{abstract}

Keywords

Primary aldosteronism; resistant hypertension; diagnosis; aldosterone; renin

\author{
Unidade de Suprarrenal, \\ Endocrinologia do \\ Desenvolvimento, Laboratório de \\ Hormônios e Genética Molecular - \\ LIM42, Divisão de Endocrinologia \\ e Metabologia, Hospital das \\ Clínicas, Faculdade de Medicina \\ da Universidade de São Paulo \\ (HCFMUSP), São Paulo, SP, Brasil \\ ${ }^{2}$ Instituto do Câncer do Estado \\ de São Paulo (Icesp), FMUSP, \\ São Paulo, SP, Brasil
}

Correspondence to: Madson Q. Almeida

Unidade de Suprarrenal,

Laboratório de Hormônios e Genética Molecular LIM-42,

Hospital das Clínicas,

Faculdade de Medicina,

Universidade de São Paulo

Av. Dr. Enéas de Carvalho Aguiar, 155,

$2^{\circ}$ andar, Bloco 6

05403-900 - São Paulo, SP, Brasil

madsonalmeida@usp.br

Received on Sept/11/2016

Accepted on May/4/2017

DOI: 10.1590/2359-3997000000274
$\mathrm{H}_{8}^{\mathrm{s}}$ ypertension(HTN) affects between 10 to $40 \%$ of the general population and is the leading risk fator for premature death in the world $(1,2)$. Robust experimental and clinical evidence implicates mineralocorticoids in the pathogenesis of HTN (3). Most monogenic forms of HTN in humans can be associated to defects in renal sodium balance (4). Several studies demonstrated that elevated aldosterone levels are predictors of adverse outcome in HTN (5), heart failure (6,7), myocardial infarction (8), and renal insufficiency (9).

Primary aldosteronism (PA) is the most common form of secondary HTN with an estimated prevalence of $4 \%$ of hypertensive patients in primary care and around 10\% of referred patients $(10,11)$. PA is particularly common in patients with resistant HTN, with a prevalence of 14 to $21 \%(12,13)$. Resistant HTN is defined as office, or clinic, systolic blood pressure (BP) of $\geq 140 \mathrm{mmHg}$, diastolic BP of $\geq 90 \mathrm{mmHg}$, or an elevation of both, on at least 3 antihypertensive medications from different drug classes, preferably including a diuretic (14).
PA is the most common curable form of HTN. Because of the adverse cardiovascular effects of excess aldosterone that are independent of high BP levels, patients with PA have higher cardiovascular morbidity and mortality than age- and sex-matched patients with essential HTN and the same degree of BP elevation $(15,16)$ (Table 1$)$.

Table 1. Cardiovascular and metabolic complications in primary aldosteronism (PA) compared to essential hypertension (EH)

\begin{tabular}{lccc}
\hline & PA (\%) & EH (\%) & $\boldsymbol{p}$ \\
\hline Cardiovascular events & & & \\
$\quad$ Atrial fibrilation (17) & 3.9 & 1.1 & 0.001 \\
$\quad$ Coronary artery disease (17) & 5.7 & 2.8 & 0.03 \\
Heart failure (17) & 4.1 & 1.2 & 0.003 \\
$\quad$ Nonfatal myocardial infarction (17) & 4.4 & 1.7 & 0.01 \\
$\quad$ Stroke (18) & 7.4 & 3.5 & 0.006 \\
Metabolic alterations & & & \\
$\quad$ Metabolic syndrome (19) & 41.1 & 29.6 & 0.05 \\
$\quad$ Abnormal glucose metabolism (20) & 22.4 & 16.8 & 0.04 \\
\hline
\end{tabular}

"Meta analysis. 
PA is characterized by an autonomous aldosterone production which is inappropriately high for sodium state and is not regulated by angiotensina II or plasma potassium concentrations. This autonomous aldosterone production causes sodium retention, plasma renin supression, HTN, cardiovascular damage, and increased potassium excretion, leading to variable degree of hypokalemia (21). In the largest study of PA prevalence, hypokalemia was identified in $48 \%$ of aldosterone-producing adenomas (aldosteronomas) and in $17 \%$ of idiopathic hyperaldosteronism (bilateral adrenal hyperplasia) (22). The frequency of PA subtypes and hypokalemia in different cohorts depends on whether PA is routinely screened among hypertensive patients and if adrenal vein sampling (AVS) is available in the specialized center. In general, aldosteroneproducing adenomas (APAs) account for around 40\% and idiopathic hyperaldosteronism for around 60\% of PA cases. APAs are small benign tumors $(1-3 \mathrm{~cm})$ originating from the glomerulosa zone, but in few cases can be smaller than $1 \mathrm{~cm}$ and diagnosed only if the AVS shows a lateralized aldosterone production. If a patient with PA has an adrenal tumor larger than $4 \mathrm{~cm}$, we should consider the rare possibility of an aldosterone-producing adrenal carcinoma. Other rare causes of PA, accounting for less than $1 \%$, are unilateral adrenal hyperplasia and familial PA, which is discussed elsewhere in this review. In our cohort of 104 PA cases from the Clinics Hospital of Sao Paulo University Medical School, aldosteronomas were diagnosed in $79 \%$ and idiopathic hyperaldosteronism in $20 \%$ of our cases, suggesting that idiopathic hyperaldosteronism is probably underdiagnosed at our institution. Familial PA type I (glucocorticoid remediable form of PA) was diagnosed in a single patient. Hypokalemia was identified in $79 \%$ of patients with aldosteronomas and in $68 \%$ of those with idiopathic hyperaldosteronism. The case detection of $\mathrm{PA}$ is recommended in the conditions listed in Table 2, as addressed in the 2016 Endocrine Society Clinical Practice Guideline of PA (23).

\section{SCREENING AND CASE CONFIRMATION}

The aldosterone-to-renin ratio is the most sensitive test that screens for PA. Immunometric assays can be employed to measure renin either by testing for plasma renin activity (PRA) or for direct renin concentration (DRC). Currently, most comercial kits measure direct renin concentration; however, the aldosterone-to-renin ratios used in PA screening were determined using PRA. Because the aldosterone-to-renin ratio is more dependent on renin, assays should be sensitive enough to detect PRA levels of $0.2-0.3 \mathrm{ng} / \mathrm{mL} / \mathrm{h}$ (or DRC of $2 \mathrm{mU} / \mathrm{L}$ ). When measuring plasma aldosterone (A) in $\mathrm{ng} / \mathrm{dL}$ and PRA in $\mathrm{ng} / \mathrm{mL} / \mathrm{h}$, the most commonly adopted A/PRA cut-off for PA screening is 30 (21). In the presence of low renin levels, the aldosterone-torenin ratio may be elevated even when aldosterone is not high. Then, a minimum aldosterone concentration of $15 \mathrm{ng} / \mathrm{dL}$ has been proposed as part of the screening criteria. In our institution, $6 \%$ of PA patients had aldosterone levels between 12.5 and $15 \mathrm{ng} / \mathrm{dL}$. Because of that, we use the minimum aldosterone level of $12.5 \mathrm{ng} / \mathrm{dL}$ to proceed with $\mathrm{PA}$ investigation (Figure 1). In a recently introduced and already commonly used automated DRC assay (DiaSorin, LIAISON XL instrument), we can use the conversion factor of $12(\mathrm{DRC} / 12=\mathrm{PRA})$ to calculate the $\mathrm{A} / \mathrm{PRA}$ ratio (21). A recent study validated this automated chemiluminescent assay for DRC and aldosterone. Using DRC $(\mathrm{mU} / \mathrm{L})$, the A/DRC ratio of $2.1 \mathrm{had}$ a sensitivity of $92 \%$, a specificity of $92 \%$, and negative predictive value of $99 \%$ to $\mathrm{PA}$ diagnosis. When using the A/DRC of 3.3 , sensitivity was $84 \%$ and specificity was $96 \%$ for PA diagnosis (24). Although we need more studies to validate the aldosterone-to-renin ratio using

Table 2. Recommendations for primary aldosteronism (PA) screening in clinical setting

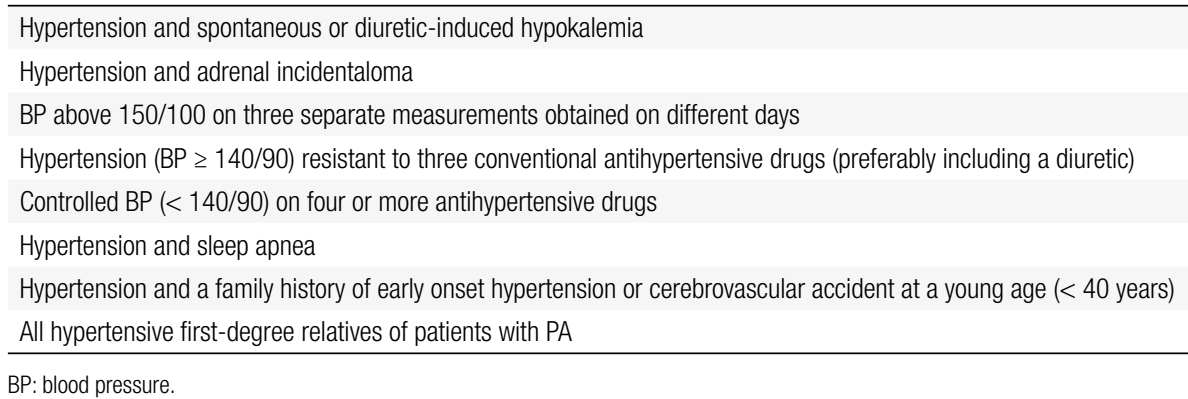


DRC, it seems that A/DRC between 2 and 3 should be equivalent to A/PRA ratio of 30 .

Positive screening for primary aldosteronism: Aldosterone $\geq 12 \mathrm{ng} / \mathrm{dL}$ and $A / D R C$ ratio $\geq 2.5$ or A/PRA ratio $\geq 30$ (correct low $\mathrm{K}^{+}$)

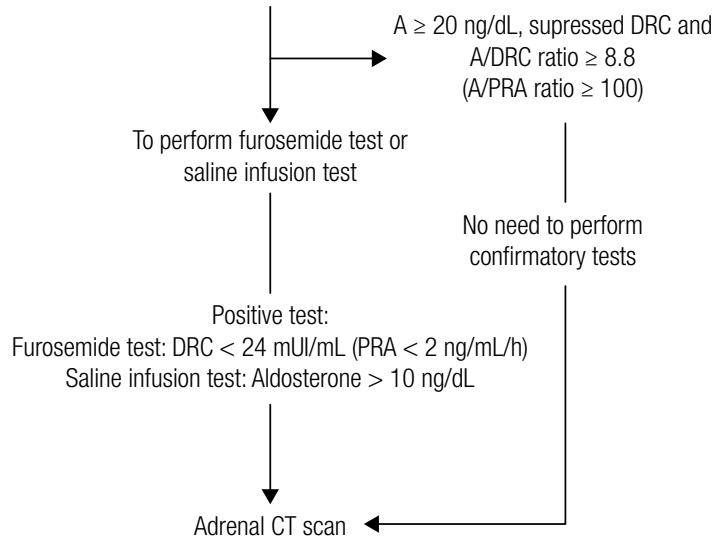

Figure 1. Algorithm for the detection and confirmation of primary aldosteronism.

A: aldosterone; DRC: direct renin concentration; PRA: plasma renin activity; CT: computed tomography.

In order to increase sensitivity, aldosterone and renin samples should be collected in the morning after patients have been out of bed for at least 2 hours, and usually after they have been seated for 5-15 minutes. Ideally, patients should have unrestricted dietary salt intake before testing and should be potassium-replete (21). It should be emphasized that mineralocorticoid antagonists (spironolactone or eplerenone) and other types of diuretics should be withdrawn for at least 4 weeks before testing. When it is not clinically possible to withdraw mineralocorticoid antagonists or diuretics, suppressed renin levels associated with high aldosterone levels strongly suggest a PA diagnosis.

In many cases (excluding mineralocorticoid antagonist or diuretic treatment), the aldosteroneto-renin ratio can be confidently interpreted despite the effect of continued medications, thus avoiding delay and allowing the patient to proceed directly to confirmatory testing. Very often, a washout of all interfering antihypertensive medications is not feasible in patients with severe HTN. Then, the aldosterone-torenin ratio should be interpreted in light of the potential confounding factors (Table 3) (21). In premenopausal ovulating women, false positives can occur during the luteal phase, but only if renin is measured as DRC. Similarly, the use of oral contraceptives is associated with a false positive screening for PA, but only if renin is measured as DRC (25). Then, if premenopausal women have a positive screening for PA, we can repeat the test without oral contraceptives (if is the case) or during the follicular phase, or even proceed with confirmatory testing.

Table 3. Factors that may interfere with screening for primary aldosteronism

\begin{tabular}{lccc}
\hline & Aldosterone & DRC & $\begin{array}{c}\text { Aldosterone/ } \\
\text { DRC }\end{array}$ \\
\hline $\begin{array}{l}\text { 3-Adrenergic blockers } \\
\text { Central agonists }\end{array}$ & $\downarrow$ & $\downarrow$ & $\uparrow(F P)$ \\
(clonidine, & $\downarrow$ & $\downarrow$ & $\uparrow(F P)$ \\
$\alpha$-methyldopa) & & & \\
$\begin{array}{l}\text { Diuretics } \\
\text { Ca }{ }^{2} \text { blockers (DHPs) }\end{array}$ & $\rightarrow \uparrow$ & $\uparrow$ & $\downarrow(F N)$ \\
ACE inhibitors, ARBs & $\downarrow$ & $\uparrow$ & $\downarrow(F N)$ \\
Advancing age & $\downarrow$ & $\downarrow$ & $\downarrow(F N)$ \\
Premenopausal women & $\rightarrow \uparrow$ & $\downarrow$ & $\uparrow(F P)$ \\
(vs. Male) & & & $\uparrow(F P)$ \\
\hline
\end{tabular}

DRC: direct renin concentration; FP: false positive; FN: false negative; ARBs: angiotensin II type 1 receptor blockers; DHP: dihydropyridines.

In the Endocrine Division of the Clinics Hospital of University of Sao Paulo Medical School, we follow the algorithm for detection and confirmation of PA shown in Figure 1. First, potassium levels should be normal to adequately interpret the aldosterone-to-renin ratio. If a patient has aldosterone levels $\geq 12.5 \mathrm{ng} / \mathrm{dL}$ and $\mathrm{A} /$ PRA $(\mathrm{ng} / \mathrm{mL} / \mathrm{h})$ ratio $\geq 30$ or $\mathrm{A} / \mathrm{DRC}(\mathrm{mU} / \mathrm{L})$ ratio $\geq 2.5$, the PA screening is considered positive. In our cohort, all PA patients had the A/PRA ratio above 30 or A/DRC ratio above 2.5 when DRC was determined.

As mentioned before, we always recommend the withdrawn of mineralocorticoid antagonists and diuretics for at least 4 weeks when clinically feasible. Otherwise, we perform the PA screening with all other antihypertensive medications. If aldosterone levels are $<20 \mathrm{ng} / \mathrm{dL}$ and PRA or DRC are not suppressed (but within the lower normal range), we recommend the replacement of the antihypertensive medications with verapamil, hydralazine, and $\alpha-1$ blockers (doxazosin or prazosin). Clonidine can be added if a fourth medication is needed.

If a patient has aldosterone levels $\geq 20 \mathrm{ng} / \mathrm{dL}$ and suppressed PRA or DRC levels, there is no need for further confirmatory testing and we can proceed with a computed tomography (CT) scan to investigate PA etiology (26). In other cases, we perform confirmatory testing to exclude false positives (Figure 1). There are several confirmatory tests and the current literature 
does not identify a "gold standard" confirmatory test for PA (21). Specialized centers choose their preferred confirmatory test based on their own experience. We suggest starting case confirmation with the furosemide test. In our experience, the furosemide test had an accuracy higher than $90 \%$ in diagnosing PA. If the furosemide test is not conclusive and the patient doesn't have contraindication for sodium loading, we perform the saline infusion test. The main problem with the saline infusion test is the volume overload in a short period performed in patients with severe/ resistant HTN or congestive heart failure. Currently, we only use the captopril test as a first option in patients with very impaired renal function, because of its too low reproducibility. Before performing any confirmatory test, we should check to see if potassium levels are normal and should correct hypokalemia. Below is detailed description of the three confirmatory tests mentioned above:

- Furosemide test: Patients receive furosemide $40 \mathrm{mg}$ iv and stay in an upright position for 2h, starting at 8-9.30 AM. Blood samples for PRA or DRC, aldosterone, and potassium are drawn at time zero and after $2 \mathrm{~h}$. PRA $<2$ $\mathrm{ng} / \mathrm{mL} / \mathrm{h}($ or $\mathrm{DRC}<24 \mathrm{mU} / \mathrm{L})$ confirms the PA diagnosis (26). Although patients with essential hypertension can present low renin levels, plasma renin activity (PRA) increases above $2 \mathrm{ng} / \mathrm{mL} / \mathrm{h}$ after furosemide injection.

- Saline infusion test: Patients stay in the recumbent position for at least $\mathrm{l} \mathrm{h}$ before and during the infusion of $2 \mathrm{~L}$ of $0.9 \%$ saline iv over $4 \mathrm{~h}$, starting at 8-9.30 AM. Blood samples for aldosterone, PRA or DRC, and potassium are drawn at time zero and after $4 \mathrm{~h}$. Aldosterone levels $>10 \mathrm{ng} / \mathrm{dL}$ confirm the diagnosis of $\mathrm{PA}$, and aldosterone $<5 \mathrm{ng} / \mathrm{dL}$ excludes the diagnosis. Aldosterone levels between 5 and 10 $\mathrm{ng} / \mathrm{dL}$ are considered indeterminate, although a cutoff of $6.8 \mathrm{ng} / \mathrm{dL}$ has been found to have the best trade-off between sensitivity and specificity (27).

- Captopril test: Patients receive $50 \mathrm{mg}$ of captopril orally after sitting or standing for at least $\mathrm{l} \mathrm{h}$. Blood samples are drawn for measurement of PRA or DRC, plasma aldosterone, and cortisol at time zero and at $\mathrm{lh}$ and $2 \mathrm{~h}$ after captopril, with the patient remaining seated during this period. Plasma aldosterone is normally suppressed by captopril (> 30\%). In patients with PA, aldosterone level remains elevated and renin remains suppressed (26). APAs can be abnormally regulated by ACTH. Then, if cortisol levels decrease during the test, we should diminish the percentage of cortisol variation from aldosterone variation to analyze only the captopril effect.

\section{SUBTYPE CLASSIFICATION}

All patients with PA should undergo adrenal CT as the initial study in subtype testing to exclude adrenocortical carcinoma. Magnetic resonance imaging has no advantage over CT in subtype evaluation of PA (21). Proper distinction between APAs and bilateral hyperplasia is crucial, because the former is treated by adrenalectomy and the latter by mineralocorticoid receptor antagonists. For the diagnosis of these two subtypes, adrenal CT scan or bilateral adrenal vein sampling (AVS) is used. Adrenal CT has several limitations, because its accuracy for diagnosing APAs is limited. CT scan can reveal normal-appearing adrenals, but the patient can have a very small APA below the detection limit of CT. Moreover, nonfunctioning unilateral adrenal adenomas are not uncommon, especially in older patients (> age 40 years), and an APA can be incorrectly diagnosed in a patient with bilateral adrenal hyperplasia and normal-appearing adrenal (28). In a different situation, bilateral adrenal nodules might be interpreted as bilateral hyperplasia on the basis of CT findings, but the patient can have an APA and an adrenal incidentaloma in the contralateral side (Figure 2).

The 2016 Endocrine Society Clinical Practice Guideline of PA recommends not to perform AVS in younger patients (< 35 years) with spontaneous hypokalemia, marked aldosterone excess, and unilateral adrenal lesions with radiological features consistent with a cortical adenoma on adrenal CT scan (21). In this situation, the probability of an adrenal incidentaloma is very low. At our institution, we don't consider the age of PA diagnosis to indicate AVS but the age of HTN diagnosis, because the median time of HTN before PA diagnosis is 14 years. In other words, most of patients with APA had been diagnosed with HTN before 35 or 40 years, but PA was only diagnosed after 40 years. This trend reflects the fact that PA screening is very delayed in our country. Based on this observation, we do not recommend AVS in patients with severe $\mathrm{PA}(\mathrm{A} \geq 20 \mathrm{ng} / \mathrm{dL}$, hypokalemia and suppressed DRC or PRA) with HTN diagnosed before 40 years and unilateral adrenal lesion $(>1 \mathrm{~cm})$ and normal contralateral adrenal on adrenal CT scan. 
In a recent randomised controlled trial (SPARTACUS TRIAL), patients with PA were randomly assigned to undergo either adrenal CT or AVS to determine the presence of APA (with subsequent treatment consisting of adrenalectomy) or bilateral adrenal hyperplasia (subsequent treatment with mineralocorticoid receptor antagonists) (29). Of the 184 patients that completed follow-up, 92 received CT-based treatment (46 adrenalectomy and 46 mineralocorticoid receptor antagonist) and 92 received AVS-based treatment (46 adrenalectomy and 46 mineralocorticoid receptor antagonist). The persistence of PA was higher in patients who received CT-based treatment (20\%) compared to patients who received AVS-based treatment (11\%), but this difference was not statistically significant (29). This important trial showed that AVS should not be recommended for all PA patients, but it did not rule out the importance of AVS in PA subtype classification, particularly in cases with bilateral lesions or normalappearing adrenal. Although the SPARTACUS trial is the first randomized study to compare CT- vs. AVS- based approaches, we need to reanalyze this issue in larger cohorts.

AVS should be performed under a condition of suppressed renin, because increased renin levels by diuretics or mineralocorticoid antagonists can lead to the stimulation of the contralateral adrenal to an APA, and unilateral PA might be misclassified as bilateral. Therefore, it is important to check if DRC or PRA is suppressed before performing AVS. An experienced radiologist is required to perform AVS. AVS includes catheterization and collecting blood samples from the right and left adrenal veins and from the inferior vena cava to measure cortisol and aldosterone. The right adrenal vein may be especially difficult to catheterize because it is short and enters the inferior vena cava at an acute angle (30). We recently started to measure serum cortisol during the AVS procedure to evaluate the successful catheterization of the right adrenal vein. Currently, our rate of successful catheterization is $80 \%$. This strategy has been previously employed to improve successful catheterization (31).

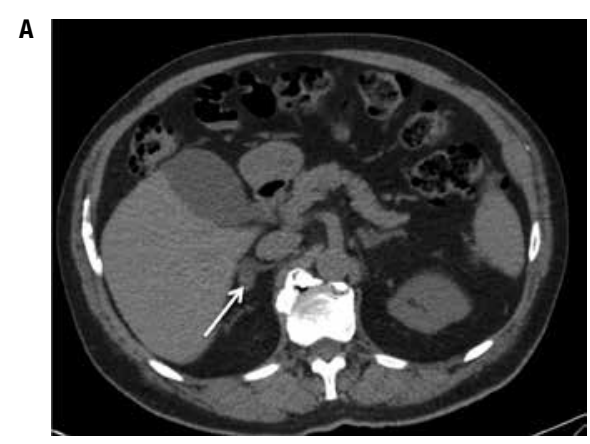

Right adrenal vein

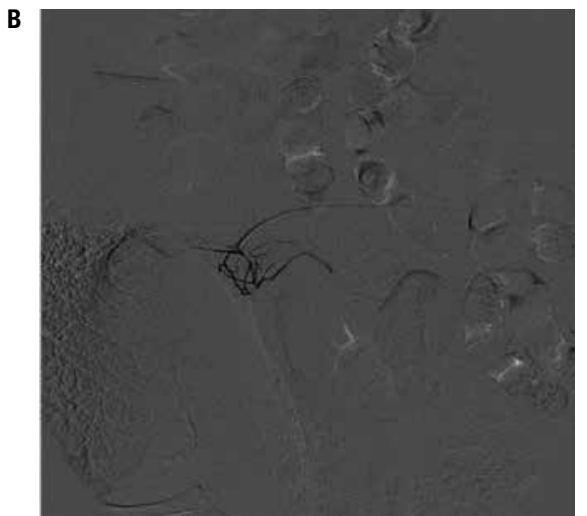

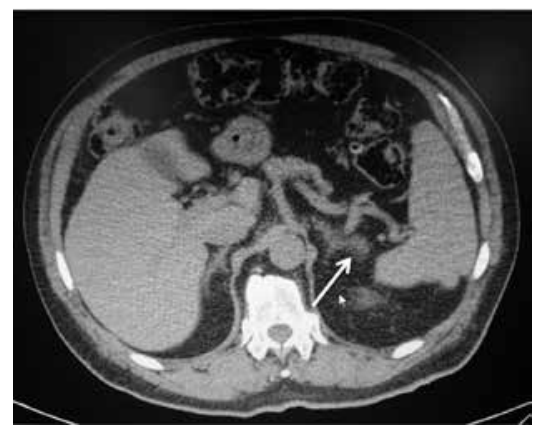

Left adrenal vein

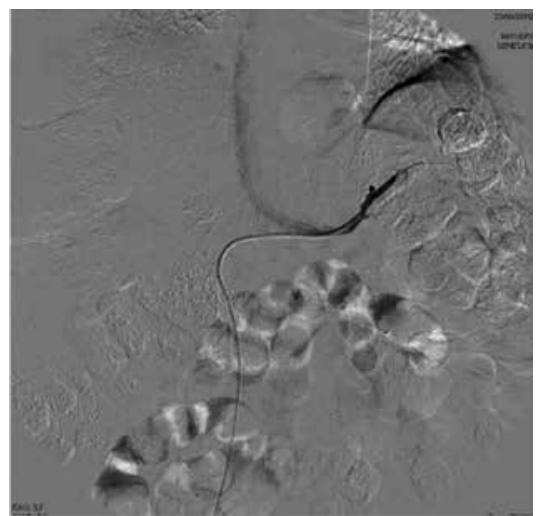

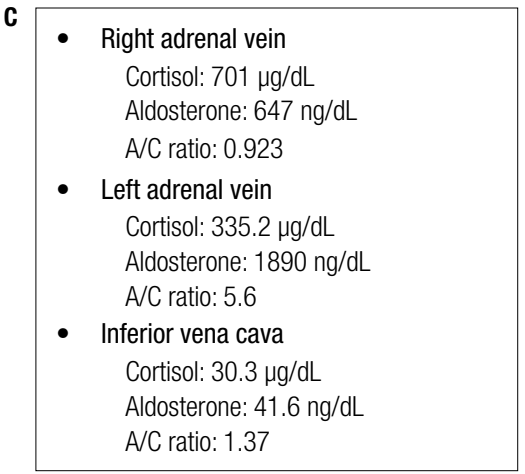

- Cortisol right adrenal vein/Inferior vena cava: 23.1

- Cortisol left adrenal vein/Inferior vena cava: 11.06

- $\quad \mathrm{A} / \mathrm{C}$ Left/Right (lateralization): 6.1

- $\quad A / C$ Right adrenal vein/Inferior vena cava (contralateral supression) $=0.67$

Figure 2. A male patient, 69 years, with resistent hypertension for 30 years, diagnosed with PA. Hormonal data: aldosterone $(A)=24.4 \mathrm{ng} / \mathrm{dL} ; \mathrm{DRC}<$ $1.6 \mathrm{mU} / \mathrm{mL}$ (PRA 0.13); A/DRC ratio = 15.25; A/PRA ratio = 188. (A) Adrenal CT showing a $1.8 \mathrm{~cm}$ nodule at right adrenal gland and a $1.2 \mathrm{~cm}$ nodule at left adrenal gland. (B) Fluoroscopic imaging from AVS. (C) Analysis of AVS sampling showed lateralization of aldosterone production to the left side. Then, this patient had an adrenal incidentaloma on the right side and an aldosterone-producing adenoma on the left side. The patient underwent left adrenal adrenalectomy and had biochemical cure of PA.

A: aldosterone; C: cortisol; A/C: aldosterone to cortisol ratio; PA: primary aldosteronism; AVS: adrenal vein sampling. 
We perform AVS under cosyntropin infusion with sequential bilateral AVS. The use of continuous cosyntropin infusion during AVS minimizes stressinduced fluctuations in aldosterone secretion (since aldosterone can be regulated by ACTH in APAs). In addition, the cosyntropin infusion maximizes the gradient of cortisol from adrenal vein to inferior vena cava, confirming the successful sampling of the adrenal vein (32). To determine the aldosterone lateralization and contralateral suppression, we use the cortisolcorrected aldosterone ratio (aldosterone divided by cortisol level in its respective vein; A/C) to correct for dilution effects. To evaluate if the nondominant adrenal (not oversecreting aldosterone) is suppressed, we calculate the contralateral suppression (defined as the $\mathrm{A} / \mathrm{C}$ ratio from the low-side to inferior vena cava). See the AVS protocol in the Clinics Hospital of Sao Paulo University Medical School below: 1) Dilute cosyntropin $(250 \mu \mathrm{g})$ in $250 \mathrm{~mL}$ of saline $0.9 \%$ and start $50 \mathrm{~mL} / \mathrm{h}$ (30 min before AVS); 2) Collect blood for aldosterone and cortisol measurements from right and left adrenal veins and from inferior vena cava; 3 ) Determine cortisol ratio between adrenal veins and the periphery (if the ratio is $>5$ on each side, the catheterization was successful); 4) Determine A/C ratio to evaluate lateralization. We should calculate the $\mathrm{A} / \mathrm{C}$ ratio from high-side to low-side. A ratio of more than 4:1 indicates unilateral aldosterone excess. A ratio of less than 3:1 suggests bilateral aldosterone hypersecretion. With these cutoffs, AVS for detecting unilateral aldosterone hypersecretion has a sensitivity of $95 \%$ and specificity of $100 \%$; 5 ) If the lateralization ratio is between $3: 1$ and $4: 1$, the AVS is undetermined. But if contralateral suppression is $<0.67$, it suggests a lateralization to the higher side (33). In Figure 2, we demonstrate a clinical case in which AVS was recommended and very useful in the subtype definition.

If a patient with $\mathrm{PA}$ has an $\mathrm{APA} \geq 2.5 \mathrm{~cm}$ or macronodular bilateral hyperplasia, screening for subclinical Cushing is recommended: $1 \mathrm{mg}$ dexamethasone suppression test, ACTH, 24h urinary free cortisol, salivar cortisol and dehydroepiandrosterone sulfate.

\section{FAMILIAL PRIMARY ALDOSTERONISM}

In patients with an onset of confirmed PA earlier than 20 years of age, and in those who have a family history of PA or stroke at a young age ( $<40$ years), the Endocrine
Society Clinical Practice Guideline of PA suggests genetic testing for familial PA type I (glucocorticoid remediable aldosteronism) and type III (caused by KCNJ5 germline mutations) (21) (Table 4).

Table 4. Familial forms of primary aldosteronism

\begin{tabular}{|c|c|c|c|}
\hline & Type I & Type III & Type III \\
\hline Cause & $\begin{array}{l}\text { Hybrid } \\
\text { CYP11B1/ } \\
\text { CYP11B2 }\end{array}$ & Unknown & $\begin{array}{c}\text { Germline } \\
\text { KCNJ5 }\end{array}$ \\
\hline Transmission & $\begin{array}{l}\text { Autosomal } \\
\text { dominant }\end{array}$ & $\begin{array}{l}\text { Autosomal } \\
\text { dominant }\end{array}$ & $\begin{array}{l}\text { Autosomal } \\
\text { dominant }\end{array}$ \\
\hline Genetic diagnosis & Long PCR & No & KCNJ5 sequencing \\
\hline $\begin{array}{l}\text { Hypertension } \\
\text { onset }\end{array}$ & $\begin{array}{l}\text { Very often } \\
<20 \text { years }\end{array}$ & Adulthood & $\begin{array}{l}\text { Very often } \\
<10 \text { years }\end{array}$ \\
\hline $\begin{array}{l}\text { Hypertension } \\
\text { severity }\end{array}$ & $\begin{array}{l}\text { Severe to resistant } \\
\text { hypertension } \\
\text { (normal BP is rare) }\end{array}$ & $\begin{array}{c}\text { Stage } 1 \text { to } \\
\text { resistant } \\
\text { hypertension } \\
\text { (normal BP is not } \\
\text { often) }\end{array}$ & $\begin{array}{c}\text { Stage } 3 \text { to } \\
\text { resistant } \\
\text { hypertension }\end{array}$ \\
\hline Hypokalemia & Rare & Not often & Very often \\
\hline $\begin{array}{l}\text { Aldosterone after } \\
\text { dexamethasone }\end{array}$ & $<4 \mathrm{ng} / \mathrm{dL}$ & $>4 \mathrm{ng} / \mathrm{dL}$ & $>4 \mathrm{ng} / \mathrm{dL}$ \\
\hline Adrenal CT & Normal & $\begin{array}{l}\text { Unilateral or } \\
\text { bilateral lesions }\end{array}$ & $\begin{array}{c}\text { Bilateral } \\
\text { macronodular } \\
\text { hyperplasia }\end{array}$ \\
\hline Treatment & $\begin{array}{l}\text { Dexamethasone } \\
\text { or } \\
\text { mineralocorticoid } \\
\text { antagonist }\end{array}$ & $\begin{array}{c}\text { Unilateral } \\
\text { adrenalectomy or } \\
\text { mineralocorticoid } \\
\text { antagonist }\end{array}$ & $\begin{array}{c}\text { Bilateral } \\
\text { adrenalectomy or } \\
\text { mineralocorticoid } \\
\text { antagonist }\end{array}$ \\
\hline
\end{tabular}

BP: blood pressure. CT: computed tomography.

Familial PA type I is caused by an unequal crossover between the genes CYPIIBI (which encodes steroid $11 \alpha$-hydroxylase) and CYPIIB2 (which encodes aldosterone synthase) $(34,35)$. The resulting hybrid gene encodes an enzyme chimera with aldosterone synthase activity that is expressed in the adrenal zona fasciculata under control of ACTH instead of angiotensin II. Patients with familial PA type I show suppressed plasma aldosterone levels $(<4 \mathrm{ng} / \mathrm{dL})$ after dexamethasone $(0.5 \mathrm{mg}$ every $6 \mathrm{~h}$ during 48 or $72 \mathrm{~h}$ ) (36). Familial PA type I is treated with dexamethasone in adults $(0.125-0.25 \mathrm{mg} / \mathrm{d})$. If additional drugs are necessary to control BP or normalize renin levels, mineralocorticoid antagonist can be added $(37,38)$.

Familial PA type II is clinically and biochemically indistinguishable from sporadic forms. Prevalence was reported to be as high as $6 \%$ in a large population with PA (39). Familial PA type II is diagnosed when at least two first-degree members of the same family have confirmed PA (APA or bilateral hyperplasia). The molecular basis of FH-II is still unknown, but genetic analyses demonstrated a link with chromosome 7p22 (40). 
Familial PA type III is caused by mutations in the KCNJ5 gene encoding the potassium channel Kir 3.4, resulting in increased sodium conductance and cell depolarization (41). Familial PA type III is characterized by severe HTN in early childhood associated with PA, hypokalemia, and macronodular bilateral hyperplasia (42). Because of the HTN severity, bilateral adrenalectomy may be needed to control BP (36).

\section{TREATMENT}

Cardiovascular morbidity caused by aldosterone excess can be decreased by either unilateral adrenalectomy or mineralocorticoid antagonist (43). Overall reduction of left ventricular mass has been demonstrated to be similar in unilateral adrenalectomy or mineralocorticoid antagonist treatment at the end of a 6.4 year follow-up (44). However, a study comparing both treatments in terms of cardiovascular mortality remains to be conducted.

Unilateral laparoscopic adrenalectomy is indicated for patients with APAs. If the patient with an APA is unable or unwilling to undergo surgery, medical treatment including a mineralocorticoid antagonist is recommended. Before unilateral adrenalectomy, the patient should be treated with mineralocorticoid antagonist in order to normalize potassium levels and renin levels, avoiding hyporeninemic hypoaldosteronism in the postoperative period. In addition, we should measure sodium, potassium, aldosterone, and renin levels in the first week after surgery to monitor treatment response. After unilateral adrenalectomy, HTN is cured in about $50 \%$ (range of $35-80 \%$ ) of patients with APA (21). Primary HTN in first-degree relatives and a longer duration of HTN before diagnosing PA are associated with low rates of HTN cure after unilateral adrenalectomy $(45,46)$.

Bilateral hyperplasia should be treated with mineralocorticoid antagonist (spironolactone or eplerenone). In Brazil, only spironolactone is available for treatment. Spironolactone $(50-400 \mathrm{mg} / \mathrm{d})$ has been the agent of choice in the medical treatment of $\mathrm{PA}$, reducing BP levels as well as the need for antihypertensive drugs (47). The starting dose for spironolactone is $50 \mathrm{mg}$ in a single dose. The dose should be increased by $50 \mathrm{mg}$ each 3-4 weeks. During treatment, we aim to control blood pressure and to normalize potassium and renin levels.

Since spironolactone antagonizes androgen receptor and inhibits androgen production, it promotes dose-dependent gynecomastia and loss of libido in men. In females, spironolactone can cause menstrual irregularities and breast tenderness and enlargement (48). To avoid or decrease side effects, amiloride or a small dose of thiazide diuretic can be used to avoid a higher dose of spironolactone. In patients with stage III chronic kidney disease or in older patients, mineralocorticoid antagonist should be administered with caution because of the risk of hyperkalemia and worsening renal function.

Eplerenone is a selective mineralocorticoid antagonist without antiandrogen effects and, therefore, is less associated with endocrine side effects and given twice daily (49). Despite its better tolerability, eplerenone has a higher cost and can be less effective than spironolactone to lower BP in the medical treatment of PA (50). In the next years, it is likely that new mineralocorticoid antagonists and selective aldosterone synthase inhibitors will be available to treat $\mathrm{PA}(2 \mathrm{l})$.

Disclosure: no potential conflict of interest relevant to this article was reported.

\section{REFERENCES}

1. Murray CJ, Lopez AD. Measuring the global burden of disease. $\mathrm{N}$ Engl J Med. 2013;369(5):448-57.

2. Egan BM, ZhaoY, Axon RN. US trends in prevalence, awareness, treatment, and control of hypertension, 1988-2008. JAMA. 2010;303(20):2043-50.

3. Stewart PM. Mineralocorticoid hypertension. Lancet. 1999; 353(9161):1341-7.

4. Lifton RP. Molecular genetics of human blood pressure variation. Science. 1996;272(5262):676-80.

5. Vasan RS, Evans JC, Larson MG, Wilson PW, Meigs JB, Rifai N, et al. Serum aldosterone and the incidence of hypertension in nonhypertensive persons. N Engl J Med. 2004;351(1):33-41.

6. Guder G, Bauersachs J, Frantz S, Weismann D, Allolio B, Ertl G, et al. Complementary and incremental mortality risk prediction by cortisol and aldosterone in chronic heart failure. Circulation. 2007;115(13):1754-61.

7. Tsutamoto T, Sakai H, Tanaka T, Fujii M, Yamamoto T, Wada A, et al. Comparison of active renin concentration and plasma renin activity as a prognostic predictor in patients with heart failure. Circ J. 2007;71(6):915-21.

8. Beygui $F$, Collet JP, Benoliel JJ, Vignolles N, Dumaine R, Barthelemy $\mathrm{O}$, et al. High plasma aldosterone levels on admission are associated with death in patients presenting with acute STelevation myocardial infarction. Circulation. 2006;114(24):2604-10.

9. Tylicki L, Larczynski W, Rutkowski B. Renal protective effects of the renin-angiotensin-aldosterone system blockade: from evidence-based approach to perspectives. Kidney Blood Press Res. 2005;28(4):230-42.

10. Hannemann A, Wallaschofski H. Prevalence of primary aldosteronism in patient's cohorts and in population-based studies--a review of the current literature. Horm Metab Res. 2012;44(3):157-62.

11. Plouin PF, Amar L, Chatellier G. Trends in the prevalence of primary aldosteronism, aldosterone-producing adenomas, and surgically correctable aldosterone-dependent hypertension. Nephrol Dial Transplant. 2004;19(4):774-7. 
12. Douma S, Petidis K, Doumas M, Papaefthimiou P, Triantafyllou A, Kartali N, et al. Prevalence of primary hyperaldosteronism in resistant hypertension: a retrospective observational study. Lancet. 2008;371(9628):1921-6.

13. Calhoun DA, Nishizaka MK, Zaman MA, Thakkar RB, Weissmann P. Hyperaldosteronism among black and white subjects with resistant hypertension. Hypertension. 2002;40(6):892-6.

14. Calhoun DA, Jones D, Textor S, Goff DC, Murphy TP, Toto RD, et al. Resistant hypertension: diagnosis, evaluation, and treatment: a scientific statement from the American Heart Association Professional Education Committee of the Council for High Blood Pressure Research. Circulation. 2008;117(25):e510-26.

15. Milliez P, Girerd X, Plouin PF, Blacher J, Safar ME, Mourad JJ. Evidence for an increased rate of cardiovascular events in patients with primary aldosteronism. J Am Coll Cardiol. 2005;45(8):1243-8.

16. Stowasser M, Sharman J, Leano R, Gordon RD, Ward G, Cowley D, et al. Evidence for abnormal left ventricular structure and function in normotensive individuals with familial hyperaldosteronism type I. J Clin Endocrinol Metab. 2005;90(9):5070-6.

17. Savard S, Amar L, Plouin PF, Steichen O. Cardiovascular complications associated with primary aldosteronism: a controlled cross-sectional study. Hypertension. 2013;62(2):331-6.

18. Mulatero P, Monticone S, Bertello $C$, Viola A, Tizzani D, lannaccone $A$, et al. Long-term cardio- and cerebrovascular events in patients with primary aldosteronism. J Clin Endocrinol Metab. 2013;98(12):4826-33.

19. Fallo F, Veglio F, Bertello C, Sonino N, Della Mea P, Ermani M, et al. Prevalence and characteristics of the metabolic syndrome in primary aldosteronism. J Clin Endocrinol Metab. 2006;91(2):454-9.

20. Chen W, Li F, He C, ZhuY, Tan W. Elevated prevalence of abnormal glucose metabolism in patients with primary aldosteronism: a meta-analysis. Ir J Med Sci. 2014;183(2):283-91.

21. Funder JW, Carey RM, Mantero F, Murad MH, Reincke M, Shibata H, et al.The Management of Primary Aldosteronism: Case Detection, Diagnosis, and Treatment: An Endocrine Society Clinical Practice Guideline. J Clin Endocrinol Metab. 2016;101(5):1889-916.

22. Rossi GP, Bernini G, Caliumi C, Desideri G, Fabris B, Ferri C, et al. A prospective study of the prevalence of primary aldosteronism in 1,125 hypertensive patients. J Am Coll Cardiol. 2006;48(11):2293-300.

23. Funder JW, Carey RM, Fardella C, Gomez-Sanchez CE, Mantero F, Stowasser M, et al. Case detection, diagnosis, and treatment of patients with primary aldosteronism: an endocrine society clinical practice guideline. J Clin Endocrinol Metab. 2008;93(9):3266-81.

24. Rossi GP, Ceolotto G, Rossitto G, SecciaTM, Maiolino G, Berton C, et al. Prospective validation of an automated chemiluminescencebased assay of renin and aldosterone for the work-up of arterial hypertension. Clin Chem Lab Med. 2016;54(9):1441-50.

25. Ahmed AH, Gordon RD, Taylor PJ, Ward G, Pimenta E, Stowasser $M$. Effect of contraceptives on aldosterone/renin ratio may vary according to the components of contraceptive, renin assay method, and possibly route of administration. J Clin Endocrinol Metab. 2011;96(6):1797-804.

26. Nanba K, Tamanaha T, Nakao K, Kawashima ST, Usui T, Tagami $\mathrm{T}$, et al. Confirmatory testing in primary aldosteronism. J Clin Endocrinol Metab. 2012;97(5):1688-94.

27. Rossi GP, Belfiore A, Bernini G, Desideri G, Fabris B, Ferri C, et al. Prospective evaluation of the saline infusion test for excluding primary aldosteronism due to aldosterone-producing adenoma. J Hypertens. 2007;25(7):1433-42.

28. Kloos RT, Gross MD, Francis IR, Korobkin M, Shapiro B. Incidentally discovered adrenal masses. Endocr Rev. 1995;16(4):460-84.

29. Dekkers T, Prejbisz A, Kool LJ, Groenewoud HJ, Velema M, Spiering W, et al. Adrenal vein sampling versus $C T$ scan to determine treatment in primary aldosteronism: an outcome-based randomised diagnostic trial. Lancet Diabetes Endocrinol. 2016;4(9):739-46.

30. Doppman JL, Gill JR, Jr. Hyperaldosteronism: sampling the adrenal veins. Radiology. 1996;198(2):309-12.

31. Yoneda T, Karashima S, Kometani M, Usukura M, Demura M, Sanada J, et al. Impact of New Quick Gold Nanoparticle-Based Cortisol Assay During Adrenal Vein Sampling for Primary Aldosteronism. J Clin Endocrinol Metab. 2016;101(6):2554-61.
32. Rossi GP, Barisa M, Allolio B, Auchus RJ, Amar L, Cohen D, et al. The Adrenal Vein Sampling International Study (AVIS) for identifying the major subtypes of primary aldosteronism. J Clin Endocrinol Metab. 2012;97(5):1606-14.

33. El Ghorayeb N, Mazzuco TL, Bourdeau I, Mailhot JP, Zhu PS, Therasse E, et al. Basal and Post-ACTH Aldosterone and Its Ratios Are Useful During Adrenal Vein Sampling in Primary Aldosteronism. J Clin Endocrinol Metab. 2016;101(4):1826-35.

34. Sutherland DJ, Ruse JL, Laidlaw JC. Hypertension, increased aldosterone secretion and low plasma renin activity relieved by dexamethasone. Can Med Assoc J. 1966;95(22):1109-19.

35. Lifton RP, Dluhy RG, Powers M, Rich GM, Cook S, Ulick S, et al. A chimaeric 11 beta-hydroxylase/aldosterone synthase gene causes glucocorticoid-remediable aldosteronism and human hypertension. Nature. 1992;355(6357):262-5.

36. Mulatero P, Monticone S, Rainey WE, Veglio F, Williams TA. Role of KCNJ5 in familial and sporadic primary aldosteronism. Nat Rev Endocrinol. 2013;9(2):104-12.

37. Dluhy RG, Anderson $B$, Harlin $B$, Ingelfinger J, Lifton $R$. Glucocorticoid-remediable aldosteronism is associated with severe hypertension in early childhood. J Pediatr. 2001;138(5):715-20.

38. Dluhy RG, Lifton RP. Glucocorticoid-remediable aldosteronism. J Clin Endocrinol Metab. 1999;84(12):4341-4.

39. PallaufA, Schirpenbach C,Zwermann O, FischerE, MorakM, HolinskiFeder $\mathrm{E}$, et al. The prevalence of familial hyperaldosteronism in apparently sporadic primary aldosteronism in Germany: a single center experience. Horm Metab Res. 2012;44(3):215-20.

40. Lafferty AR, Torpy DJ, Stowasser M, Taymans SE, Lin JP, Huggard $P$, et al. A novel genetic locus for low renin hypertension: familial hyperaldosteronism type II maps to chromosome 7 (7p22). J Med Genet. 2000;37(11):831-5.

41. Scholl UI, Nelson-Williams C, Yue P, Grekin R, Wyatt RJ, Dillon MJ, et al. Hypertension with or without adrenal hyperplasia due to different inherited mutations in the potassium channel KCNJ5. Proc Natl Acad Sci U S A. 2012;109(7):2533-8.

42. Geller DS, Zhang J, Wisgerhof MV, Shackleton C, Kashgarian M, Lifton RP. A novel form of human mendelian hypertension featuring nonglucocorticoid-remediable aldosteronism. J Clin Endocrinol Metab. 2008;93(8):3117-23.

43. Rossi GP, Bolognesi M, Rizzoni D, Seccia TM, Piva A, Porteri E, et al. Vascular remodeling and duration of hypertension predict outcome of adrenalectomy in primary aldosteronism patients. Hypertension. 2008;51(5):1366-71.

44. Catena C, Colussi G, Lapenna R, Nadalini E, Chiuch A, Gianfagna P, et al. Long-term cardiac effects of adrenalectomy or mineralocorticoid antagonists in patients with primary aldosteronism. Hypertension. 2007;50(5):911-8.

45. Celen O, O'Brien MJ, Melby JC, Beazley RM. Factors influencing outcome of surgery for primary aldosteronism. Arch Surg. 1996;131(6):646-50.

46. Sawka AM, Young WF, Thompson GB, Grant CS, Farley DR, Leibson $C$, et al. Primary aldosteronism: factors associated with normalization of blood pressure after surgery. Ann Intern Med. 2001;135(4):258-61.

47. Wambach G, Helber A, Bonner G, Hummerich W, Meurer KA, KaufmannW. [Spironolactone in essential hypertension associated with abnormal aldosterone regulation and in Conn's syndrome (author's transI)]. Dtsch Med Wochenschr. 1980;105(18):647-51.

48. Jeunemaitre $X$, Chatellier G, Kreft-Jais C, Charru A, DeVries $\mathrm{C}$, Plouin PF, et al. Efficacy and tolerance of spironolactone in essential hypertension. Am J Cardiol. 1987;60(10):820-5.

49. Burgess ED, LacourciereY, Ruilope-Urioste LM, Oparil S, Kleiman $\mathrm{JH}$, Krause S, et al. Long-term safety and efficacy of the selective aldosterone blocker eplerenone in patients with essential hypertension. Clin Ther. 2003;25(9):2388-404.

50. Parthasarathy HK, Menard J, White WB, Young WF, Jr., Williams $\mathrm{GH}$, Williams B, et al. A double-blind, randomized study comparing the antihypertensive effect of eplerenone and spironolactone in patients with hypertension and evidence of primary aldosteronism. J Hypertens. 2011;29(5):980-90. 